\title{
Effect of Hachimijiogan against Renal Dysfunction and Involvement of Hypoxia-Inducible Factor- $1 \alpha$ in the Remnant Kidney Model
}

\author{
Hiroshi Oka, ${ }^{1}$ Hirozo Goto, ${ }^{1}$ Keiichi Koizumi, ${ }^{2,3}$ Shin Nakamura, ${ }^{4}$ Koichi Tsuneyama, ${ }^{5}$ \\ Yue Zhou, ${ }^{2}$ Michiko Jo, ${ }^{3}$ Takako Fujimoto, ${ }^{6}$ Hiroaki Sakurai, ${ }^{2}$ Naotoshi Shibahara, ${ }^{3}$ \\ Ikuo Saiki, ${ }^{2}$ and Yutaka Shimada ${ }^{1}$ \\ ${ }^{1}$ Department of Japanese Oriental Medicine, Graduate school of Medicine and Pharmaceutical Sciences, University of Toyama, \\ 2630 Sugitani, Toyama 930-0194, Japan \\ ${ }^{2}$ Division of Pathogenic Biochemistry, Institute of Natural Medicine, University of Toyama, 2630 Sugitani, Toyama 930-0194, Japan \\ ${ }^{3}$ Department of Kampo Diagnostics, Institute of Natural Medicine, University of Toyama, 2630 Sugitani, Toyama 930-0194, Japan \\ ${ }^{4}$ Biomedical Institute, NPO Primate Agora, Inuyama, Aichi 484-0002, Japan \\ ${ }^{5}$ Department of Diagnostic Pathology, Graduate School of Medicine and Pharmaceutical Sciences, University of Toyama, 2630 Sugitani, \\ Toyama 930-0194, Japan \\ ${ }^{6}$ Faculty of Human Development, University of Toyama, 3190 Gofuku, Toyama 930-8555, Japan
}

Correspondence should be addressed to Hirozo Goto, hiro510@med.u-toyama.ac.jp

Received 2 November 2010; Accepted 7 January 2011

Copyright () 2011 Hiroshi Oka et al. This is an open access article distributed under the Creative Commons Attribution License, which permits unrestricted use, distribution, and reproduction in any medium, provided the original work is properly cited.

In chronic renal failure, hypoxia of renal tissue is thought to be the common final pathway leading to end-stage renal failure. In this study the effects of hachimijiogan, a Kampo formula, were studied with respect to hypoxia-inducible factor (HIF). Using remnant kidney rats, we studied the effects of hachimijiogan on renal function in comparison with angiotensin II receptor blocker. The result showed that oral administration of hachimijiogan for seven days suppressed urinary protein excretion and urinary 8 OHdG, a marker of antioxidant activity, equally as well as oral administration of candesartan cilexetil. In contrast, the protein volume of HIF- $1 \alpha$ in the renal cortex was not increased in the candesartan cilexetil group, but that in the hachimijiogan group was increased. In immunohistochemical studies as well, the expression of HIF-1 $\alpha$ of the high-dose hachimijiogan group increased compared to that of the control group. Vascular endothelial growth factor and glucose transporter 1, target genes of HIF- $1 \alpha$, were also increased in the hachimijiogan group. These results suggest that hachimijiogan produces a protective effect by a mechanism different from that of candesartan cilexetil.

\section{Introduction}

In recent years, it has been clear that chronic kidney disease (CKD) is an important risk factor for cardiovascular diseases and mortality and has been the focus of considerable attention [1]. The number of dialysis patients caused by endstage renal disease has been increasing worldwide, regardless of the advances in treatments, such as a protein- and sodium-restricted diet, medicines containing angiotensin II receptor blocker (ARB), and kidney transplants [2]. There are various causes for the early stage of CKD, but there is a common pathway in advanced CKD as represented by interstitial fibrosis, glomerular sclerosis, and nephron destruction [3]. The kidney is sensitive to oxygen supply and is prone to sustaining damage from hypoxia. In recent years, it has been reported that chronic hypoxia in the kidney is the final common pathway to end-stage renal failure $[4,5]$. Thus, therapeutic approaches against hypoxic injury in CKD patients are considered important for preventing the worsening of CKD. As a biological defense mechanism against tissue hypoxia, hypoxia-inducible factor (HIF), a heterodimeric transcription factor, plays an essential role. Activation of HIF stimulates numerous downstream target genes and protects tissue from hypoxia [6]. This effect is suggested by reports, which showed that cobalt ameliorates renal injury of renal disease model rats [7], and vascular 
endothelial growth factor (VEGF) enhances glomerular capillary repair and accelerates resolution of experimentally induced glomerulonephritis [8].

Hachimijiogan, which we used in this study, is a Kampo formula created more than 1800 years ago. It is composed of eight crude drugs: Cinnamomi Cortex, Aconiti Japonici Tuber, Rehmanniae Radix, Corni Fructus, Dioscoreae Rhizoma, Alismatis Rhizoma, Moutan Cortex, and Hoelen. It has been clinically used to treat many symptoms, such as lumbago, pollakisuria, cold hands and feet, nephritis, and so on [9]. In basic research, it has been reported that hachimijiogan inhibits the progression of renal dysfunction in diabetic nephropathy model rats and 5/6 nephrectomized model rats. As the mechanism of its action, reduction of uremic toxins associated with antioxidant activity and positive effects on organic change of the kidney have been reported $[10,11]$.

However, there are no studies reporting the effect of hachimijiogan on HIF. Therefore, we examined the effect of hachimijiogan in comparison to ARB, a mainstream drug for renal disorder, on renal dysfunction from the aspect of hypoxic injury using 5/6 nephrectomized rats, in which tissue hypoxia causes progression of renal failure $[12,13]$.

\section{Materials and Methods}

2.1. Materials. The bulk extract of hachimijiogan (batch number 2060007020), which is approved for medical use in Japan, was purchased from Tsumura Co. Ltd. (Tokyo, Japan). It consists of eight herbs: $6.0 \mathrm{~g}$ of Rehmanniae Radix (Rehmannia glutinosa Liboschitz var. purpurea Makino), $3.0 \mathrm{~g}$ of Corni Fructus (Cornus officinalis Siebold et Zuccarini), $3.0 \mathrm{~g}$ of Dioscoreae Rhizoma (Dioscorea japonica Thunberg), $3.0 \mathrm{~g}$ of Alismatis Rhizoma (Alisma orientale Juzepczuk), $3.0 \mathrm{~g}$ of Hoelen (Poria cocos Wolf), $3.0 \mathrm{~g}$ of Moutan Cortex (Paeonia suffruticosa Andrews), $2.5 \mathrm{~g}$ of Cinnamomi Cortex (Cinnamomum cassia Blume), $1.0 \mathrm{~g}$ of Aconiti Tuber (Aconitum carmichaeli Debeaux).

Candesartan cilexetil was obtained from Takeda Pharmaceutical Company Ltd. (Osaka, Japan).

\subsection{Three-Dimensional HPLC Analysis of Hachimijiogan.} For analysis of the components of hachimijiogan, aqueous extract $(1 \mathrm{~g})$ was extracted with $20 \mathrm{~mL}$ methanol under ultrasonication for $30 \mathrm{~min}$. The solution was filtered through a membrane filter $(0.45 \mu \mathrm{m})$ and then subjected to highperformance liquid chromatography (HPLC) analysis using a TSK-GEL ODS-80TS column $(\varphi 4.6 \times 250 \mathrm{~mm}$, Tosoh, Tokyo, Japan) with an LC 10AD pump and an SPDM10A absorbance detector. The elution solvents were (A)

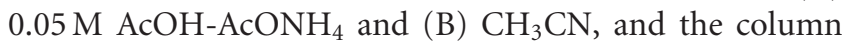
was eluted with a linear gradient of, by volume, $90 \% \mathrm{~A}$ and $10 \% \mathrm{~B}$ changing over $60 \mathrm{~min}$ to $100 \% \mathrm{~B}$. The flow rate was $1.0 \mathrm{~mL} / \mathrm{min}$, and the effluent from the column was monitored and processed into three-dimensional data by an SPD-M10A array detector. All assigned peaks were identified by comparing their UV spectral data with those of coinjected authentic samples using the Class LC-10 Version
1.62 software package (Shimadzu, Kyoto, Japan). The threedimensional HPLC profile of hachimijiogan extract is shown in Figure 1. The major components of hachimijiogan were morroniside, loganin, paeoniflorin, penta-O-galloyl glucose, benzoylmesaconine, benzoylpaeoniflorin, 16-ketoalisol A, paeonol, cinnamic acid, and cinnamaldehyde.

2.3. Animals and Treatments of Animals. All experimental procedures were performed in accordance with the standards established by the "Guide for the Care and Use of Laboratory Animals at the University of Toyama". Fifty 6-week-old male Sprague-Dawley rats were purchased from Japan SLC Inc. (Hamamatsu, Japan) and kept in an automatically controlled room (temperature about $23^{\circ} \mathrm{C}$ and humidity about 60\%) with a conventional dark/light cycle. The animals were kept in metabolic cages, and 24-hour urine samples were collected. Blood pressure was determined by tail cuff system (MK2000, Muromachi Kikai Co., Ltd., Tokyo, Japan) in a conscious state. At 7 weeks old, 40 rats underwent 5/6 nephrectomy under anesthesia with sodium pentobarbital $(50 \mathrm{mg} / \mathrm{kg}$ body weight, i.p.) by ablation of approximately $2 / 3$ of the left kidney, and then removal of the right kidney by ligation of renal artery, vein, and ureter 1 week later. After recovery from the operation (after 1 week), the $5 / 6$ nephrectomized $(5 / 6 \mathrm{Nx})$ rats were randomly divided into four groups (control and three treatment groups, $n=$ 10 /group). One more group of rats had undergone a sham operation $(n=10)$. During the experimental period, all groups were fed a standard chow. The sham and control groups were fed water, and the other three surgical groups were fed a solution of hachimijiogan extract orally at a dose of $220 \mathrm{mg} / \mathrm{kg}$ body weight/day (low-dose hachimijiogan), $660 \mathrm{mg} / \mathrm{kg}$ body weight/day (high-dose hachimijiogan), or a solution of candesartan cilexetil orally at a dose of $3 \mathrm{mg} / \mathrm{kg}$ body weight/day, respectively, by gastric gavage. These doses of hachimijiogan for rats were approximately 3 times and 10 times the human dose of hachimijiogan. After 7 days of treatment, the rats were sacrificed, and blood samples were obtained. The kidneys were removed from each rat, frozen quickly, and kept at $-80^{\circ} \mathrm{C}$ until analysis.

2.4. Analysis of Serum and Urine Samples. Serum levels of Albumin were determined by SRL, Inc. (Tokyo, Japan). Serum levels of urea nitrogen (BUN) and creatinine (sCre) were determined using commercial kits (BUN Kainos and CRE-EN Kainos purchased from Kainos Laboratories, Inc., Tokyo, Japan). Urinary protein (u-Pro) excretion levels were determined using commercial reagents (Micro TP-test, Wako Pure Chemical, Osaka, Japan). Creatinine clearance (Ccr) was calculated on the basis of urinary creatinine, serum creatinine, urine volume, and body weight using the following equation: $\mathrm{Ccr}(\mathrm{mL} /(\mathrm{kg}$ body weight $) / \mathrm{min})$ $=\{$ urinary Cre $(\mathrm{mg} / \mathrm{dL}) \times$ urine volume $(\mathrm{mL}) /$ serum Cre $/(\mathrm{mg} / \mathrm{dL})\} \times\{1,000 /$ body weight $(\mathrm{g})\} \times\{1 / 1,440(\mathrm{~min})\}$. 8 -Hydroxy-deoxyguanosine (8-OHdG) content in 24-hour urine samples was measured by ELISA kit (8-OHdG Check, JaICA, Nikken SEIL Co., Shizuoka, Japan). 


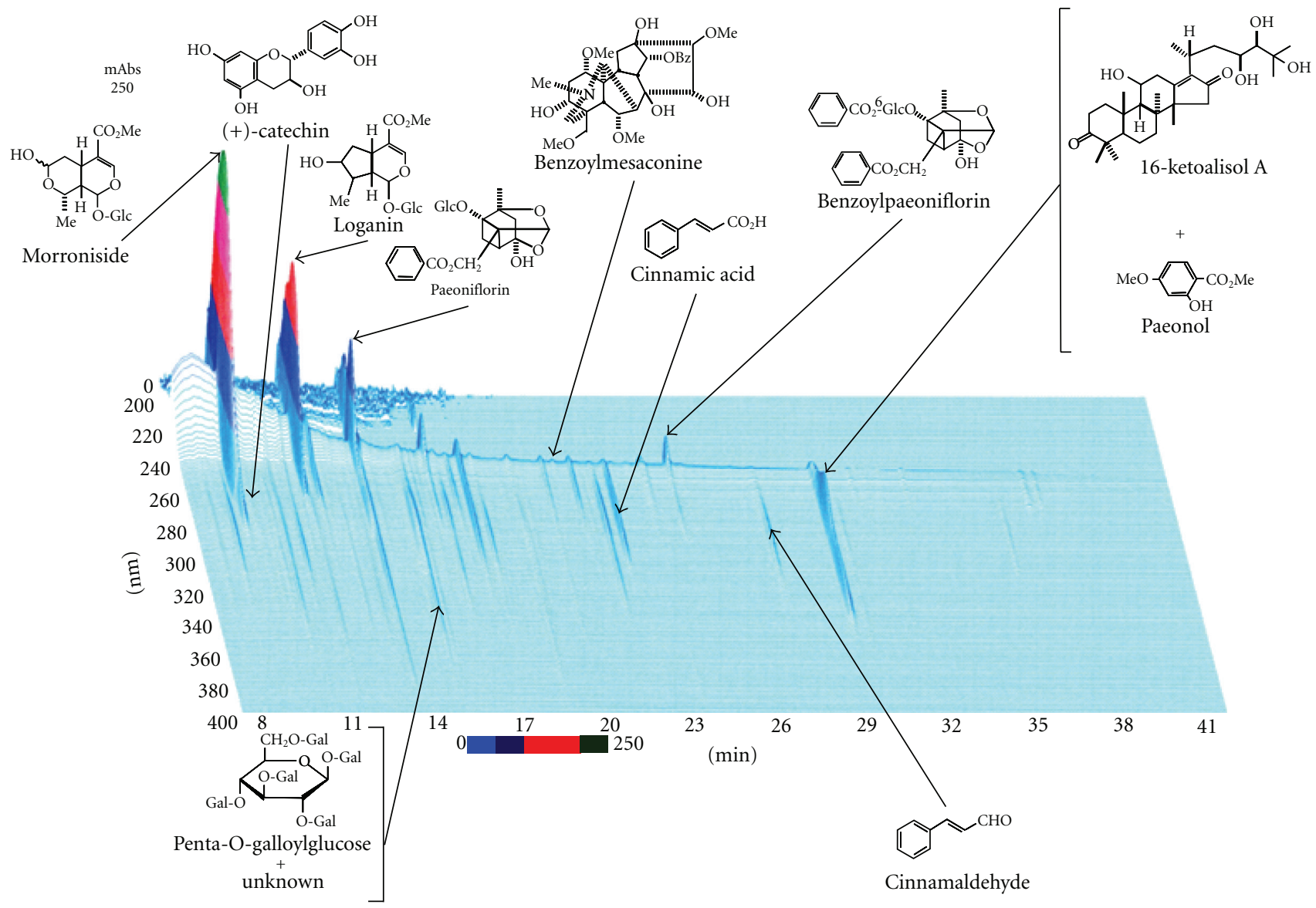

Figure 1: Chemical profile of hachimijiogan analyzed by three-dimensional HPLC.

2.5. Real-Time RT-PCR. Total RNA was prepared using the RNeasy Mini kit (QIAGEN, Valencia, CA, USA). First-strand cDNA was synthesized by SuperScript II reverse transcriptase (Invitrogen, Carlsbad, CA, USA). cDNA was amplified quantitatively using SYBR Premix Ex Taq (TaKaRa-Bio, Otsu, Japan). The primer sequences are summarized in Table 1 . Real-time quantitative RT-PCR was performed using an ABI Prism 7300 sequence detection system (Applied Biosystems, Foster City, CA, USA). All data were normalized to $\beta$-actin mRNA.

2.6. Protein Preparation and Western Blotting. The cortex was dissected from the frozen kidney and homogenized in buffer A ( $10 \mathrm{mM}$ HEPES $\mathrm{pH} 7.9,10 \mathrm{mM} \mathrm{KCl}, 0.1 \mathrm{mM}$ EDTA, $0.1 \mathrm{mM}$ EGTA, $1 \mathrm{mM}$ DTT, $1 \mathrm{mM}$ PMSF, $20 \mathrm{mM} \beta$ glycerophosphate, $0.1 \mathrm{mM}$ sodium orthovanadate, $10 \mu \mathrm{g} / \mathrm{mL}$ aprotinin, and $10 \mu \mathrm{g} / \mathrm{mL}$ leupeptin) and chilled on ice for 15 min. Next, $25 \mu \mathrm{L}$ of $10 \%$ Nonidet P-40 was added and the suspension was vigorously vortexed for $10 \mathrm{~s}$ and kept on ice for $5 \mathrm{~min}$. The nuclear pellets were washed with $100 \mu \mathrm{L}$ of buffer $\mathrm{A}$ and suspended in $50 \mu \mathrm{L}$ of buffer $\mathrm{C}$ ( $20 \mathrm{mM}$ HEPES pH 7.9, 0.4 M NaCl, 1 mM EDTA, 1 mM EGTA, 1 mM DTT, $1 \mathrm{mM}$ PMSF, $20 \mathrm{mM} \beta$-glycerophosphate, $1 \mathrm{mM}$ sodium orthovanadate, $10 \mu \mathrm{g} / \mathrm{mL}$ aprotinin, $10 \mu \mathrm{g} / \mathrm{mL}$ leupeptin). The mixture was kept on ice for $15 \mathrm{~min}$ with frequent agitation. Nuclear extracts were prepared by centrifugation at $15,000 \mathrm{rpm}$ for $5 \mathrm{~min}$.

Kidney lysates were resolved by SDS-PAGE and transferred to Immobilon-P nylon membrane (Millipore, Bedford, MA, USA). The membrane was treated with BlockAce (DS pharma Co. Ltd., Suita, Japan) overnight at $4^{\circ} \mathrm{C}$ and probed with primary antibodies. An antibody against HIF$1 \alpha$ (H1alpha67) was purchased from Abcam (Cambridge, UK). Lamin B was used as an internal control. Antibodies against Lamin B (C-20) were purchased from Santa Cruz Biotechnologies (Santa Cruz, CA, USA). Enhancer solutions (Can Get Signal; Toyobo, Osaka, Japan) were used for the dilution. The antibodies were detected using horseradish peroxidase-conjugated antimouse and antigoat IgG (Dako Cytomation, Glostrup, Denmark) and visualized with the ECL system for Lamin B and ECL-plus for HIF-1 $\alpha$ (GE Healthcare, Buckinghamshire, UK).

2.7. Histology and Immunohistochemistry. Rats were deeply anesthetized by an intraperitoneal injection of pentobarbital sodium ( $50 \mathrm{mg} / \mathrm{kg}$ body weight). Kidney was rapidly excised and immediately immersed in $4 \%$ paraformaldehyde and embedded in paraffin. Sections ( $5 \mu \mathrm{m}$ thick) were routinely stained with hematoxylin and eosin. Mouse monoclonal antibody against HIF-1 $\alpha$ (H1alpha, 1:25 diluted; Novus 
TABLE 1: Sequences for RT-PCR primers.

\begin{tabular}{lcc}
\hline Genes & Forward & Reverse \\
\hline$\beta$-actin & GCCAACCGTGAAAAGATGAC & AGGCATACAGGGACAACACA \\
VEGF & TTACTGCTGTACCTCCAC & ACAGGACGGCTTGAAGATA [12] \\
Glut-1 & AGGTGTTCGGCTTAGACTC & GAAGGGCAACAGGATACAC \\
\hline
\end{tabular}

VEGF: vascular endothelial growth factor, Glut-1: glucose transporter 1.

Biologicals, Littleton, CO, USA) was used for immunohistochemical staining of kidney as previously described [14]. For detecting primary antibodies on rat tissue specimens, M.O.M. kit (Vector, Burlingame, CA, USA) was used for special blocking. Tissue sections were cut at 5 micrometers from tissue blocks and placed on slides. After deparaffinization, sections were soaked in target retrieval solution (TRS, pH 6.1, Dako Cytomation) in a nonmetal-containing plasticmade pressure cooker and irradiated in a microwave oven for 15 minutes (maximum $500 \mathrm{~W}$ ). After irradiation, sections were rinsed under running water for 2 minutes, soaked in 3\% $\mathrm{H}_{2} \mathrm{O}_{2}$ methanol solution for 5 minutes, and then soaked in 5\% BSA for 1 minute. After that, M.O.M. mouse Ig blocking reagent was applied and incubated for 1 hour. Primary antibody was diluted to a previously determined optimal concentration in M.O.M. diluent. The diluted antibody was applied to the tissue sections in a moist chamber and irradiated intermittently for 30 minutes $(250 \mathrm{~W}, 4$ seconds on, 3 seconds off). After three washes with Trisbuffered saline containing 1\% Tween (TBS-T) for 5 minutes, peroxidase-conjugated Envision kit for mouse (EnvisionPO, Envision System, Dako Cytomation) was used on the appropriate specimens in the moist chamber. Irradiation was then performed intermittently for 30 minutes as described above. After washing 5 times with TBS, the sections were immersed in DAB solution (Sigma-Aldrich, St. Louis, MO, USA) with $\mathrm{H}_{2} \mathrm{O}_{2}$ and counterstained with hematoxylin (Dako Cytomation) and mounted under coverslips.

Immunopositivity for HIF- $1 \alpha$ in the tubular cells of the cortex was counted using 18 fields per group, and the average number per field was determined.

2.8. Statistical Analysis. All values were presented as mean \pm S.D., and were analyzed by one-way analysis of variance (ANOVA) followed by Dunnett's test. $P<.05$ was accepted as statistically significant.

\section{Results}

3.1. Body Weight, Kidney Weight, Blood Pressure, and Urinary Volume. Table 2 shows the changes in body weight, kidney weight, blood pressure and urinary volume of the rats during the 1 -week experimental period. The final body weights of the $5 / 6 \mathrm{Nx}$ groups were significantly lower than that of the sham group. There were no significant differences between baseline and final body weights in the $5 / 6 \mathrm{Nx}$ groups. The remnant kidney weights among the $5 / 6 \mathrm{Nx}$ groups did not change after the 1-week treatment. Blood pressures among all groups also did not change during the 1-week treatment.
Urinary volumes of the $5 / 6 \mathrm{Nx}$ groups increased significantly compared to the sham group. There were no significant differences between baseline and final urinary volumes in the $5 / 6 \mathrm{Nx}$ groups.

3.2. Serum and Urine Biochemical Parameters. Table 3 shows the effects of hachimijiogan on serum and urine biochemical parameters. BUN levels of the control and low-dose hachimijiogan groups were significantly higher than that of the sham group. There were no significant differences in BUN levels among the four $5 / 6 \mathrm{Nx}$ groups. The s-Cre level of the control group was significantly higher than that of the sham group. There were no significant differences in sCre levels among the four $5 / 6 \mathrm{Nx}$ groups. Urinary protein excretion of the control group was significantly increased compared to that of the sham group, and those of the high-dose hachimijiogan and candesartan cilexetil groups were significantly decreased compared to that of the control group. Ccr levels of the four 5/6Nx groups were significantly decreased compared to that of the sham group. The 8-OHdG level of the control group was significantly higher than that of the sham group, and those of the low-dose hachimijiogan, high-dose hachimijiogan, and candesartan cilexetil groups were significantly lower than that of the control group.

3.3. Renal Cortical Hypoxia-Related Factors. The volumes of HIF- $1 \alpha$ protein of the four $5 / 6 \mathrm{Nx}$ groups were significantly increased compared to that of the sham group, and that of the high-dose hachimijiogan group was significantly increased compared to that of the control group (Figure 2(a)). Figures 2(b) and 2(c) show the effects of hachimijiogan on renal mRNA levels of VEGF and glucose transporter-1 (Glut-1). In the high-dose hachimijiogan group, the mRNA levels of VEGF and Glut-1 were significantly increased compared to those of the sham and control groups.

Immunohistochemical studies also showed that the expression of HIF- $1 \alpha$ of the high-dose hachimijiogan group was increased compared to that of the control group (Figures 3(a)-3(e)). There was a significantly large number of HIF$1 \alpha$-positive cells in the high-dose hachimijiogan group compared to the control group (Figure 3(f)).

\section{Discussion}

Recently, as a final common pathway of various renal diseases, attention has been focused on tubulointerstitial hypoxia. It has been reported that the hypoxia of renal tissue was caused by decreasing peritubular capillary blood flow due to renal fibrosis, abnormal production of vasoactive 
TABLE 2: Physiological data of experimental animals.

\begin{tabular}{|c|c|c|c|c|c|c|c|c|c|}
\hline \multirow[t]{2}{*}{ Group } & \multicolumn{2}{|c|}{ Body weight (g) } & \multirow[t]{2}{*}{$\begin{array}{l}\text { Kidney weight } \\
\text { (g/100g BW) }\end{array}$} & \multicolumn{2}{|c|}{$\begin{array}{l}\text { Systolic blood } \\
\text { pressure }(\mathrm{mmHg})\end{array}$} & \multicolumn{2}{|c|}{$\begin{array}{l}\text { Diastolic blood } \\
\text { pressure }(\mathrm{mmHg})\end{array}$} & \multicolumn{2}{|c|}{$\begin{array}{l}\text { Urine volume } \\
\text { (mL/day) }\end{array}$} \\
\hline & Baseline & Final & & Baseline & Final & Baseline & Final & Baseline & Final \\
\hline Sham & $297.1 \pm 11.0$ & $325.5 \pm 12.0$ & $0.311 \pm 0.04$ & $121.4 \pm 14.2$ & $113.7 \pm 10.8$ & $56.4 \pm 13.5$ & $62.6 \pm 16.2$ & $5.7 \pm 3.1$ & $6.4 \pm 2.8$ \\
\hline Control & $261.7 \pm 22.9$ & $291.6 \pm 26.4^{*}$ & $0.331 \pm 0.05$ & $124.3 \pm 5.8$ & $127.7 \pm 8.2$ & $69.7 \pm 6.7$ & $59.1 \pm 12.2$ & $20.3 \pm 6.6^{* *}$ & $21.1 \pm 6.1^{* *}$ \\
\hline LD-HJG & $253.6 \pm 17.6$ & $275.9 \pm 24.3^{*}$ & $0.341 \pm 0.03$ & $132.9 \pm 27.1$ & $130.0 \pm 17.2$ & $74.2 \pm 19.1$ & $62.8 \pm 16.2$ & $17.6 \pm 6.7^{* *}$ & $14.1 \pm 6.3^{* *}$ \\
\hline HD-HJG & $257.5 \pm 16.6$ & $295.6 \pm 16.4^{*}$ & $0.354 \pm 0.04$ & $122.8 \pm 15.6$ & $126.3 \pm 8.9$ & $70.1 \pm 17.3$ & $56.9 \pm 6.1$ & $20.6 \pm 8.3^{* *}$ & $18.0 \pm 7.1^{* *}$ \\
\hline Candesartan & $264.3 \pm 14.6$ & $276.1 \pm 16.6^{*}$ & $0.343 \pm 0.03$ & $121.4 \pm 14.6$ & $119.7 \pm 16.2$ & $70.9 \pm 15.8$ & $58.5 \pm 13.1$ & $20.2 \pm 13.4^{* *}$ & $16.5 \pm 12.8^{* *}$ \\
\hline
\end{tabular}

LD-HJG: low-dose hachimijiogan; HD-HJG: high-dose hachimijiogan.

Baseline: before drug administration; Final: after 1 week of drug administration.

Data represent mean \pm S.D. $(n=8-10) .{ }^{*} P<.05,{ }^{* *} P<.01$ versus sham group.

TABLE 3: Effect of hachimijiogan on renal functional parameters.

\begin{tabular}{lcccccc}
\hline & s-Alb $(\mathrm{mg} / \mathrm{dL})$ & BUN $(\mathrm{mg} / \mathrm{dL})$ & s-Cre $(\mathrm{mg} / \mathrm{dL})$ & u-Pro $(\mathrm{mg} /$ day $)$ & Ccr $(\mathrm{mL} / \mathrm{min} / \mathrm{kg}$ BW $)$ & 8 -OHdG $(\mathrm{ng} / \mathrm{day})$ \\
\hline Sham & $834.8 \pm 110.9$ & $15.6 \pm 1.9$ & $0.32 \pm 0.14$ & $13.01 \pm 7.29$ & $5.03 \pm 3.07$ & $325.9 \pm 106.5$ \\
Control & $887.1 \pm 67.4$ & $50.6 \pm 36.7^{* *}$ & $1.44 \pm 1.32^{* *}$ & $35.34 \pm 20.62^{* *}$ & $1.88 \pm 0.94^{* *}$ & $840.4 \pm 252.4^{* *}$ \\
LD-HJG & $895.8 \pm 25.7$ & $42.0 \pm 27.1^{*}$ & $0.93 \pm 0.59$ & $25.15 \pm 15.32$ & $2.13 \pm 1.40^{* *}$ & $582.9 \pm 201.8^{\#}$ \\
HD-HJG & $833.9 \pm 82.2$ & $39.6 \pm 25.7$ & $0.87 \pm 0.61$ & $19.73 \pm 5.41^{\#}$ & $2.11 \pm 0.86^{* *}$ & $550.9 \pm 210.5^{\#}$ \\
Candesartan & $840.8 \pm 92.5$ & $33.8 \pm 12.3$ & $0.88 \pm 0.49$ & $15.41 \pm 5.92^{\# \#}$ & $1.65 \pm 0.64^{* *}$ & $484.2 \pm 171.3^{\# \#}$ \\
\hline
\end{tabular}

LD-HJG: low-dose hachimijiogan; HD-HJG: high-dose hachimijiogan.

Data represent mean \pm S.D. $(n=8-10)$.

${ }^{*} P<.05,{ }^{* *} P<.01$ versus sham group.

${ }^{\#} P<.05,{ }^{\# \#} P<.01$ versus control group.

substance, anemia, and so on [15]. HIF, a heterogeneous nuclear ribonucleoprotein, is an important defense factor against tissue hypoxia. Activation of HIF and numerous downstream target genes protect tissues from hypoxia [6]. Under normoxic conditions, HIF- $1 \alpha$ subunit is hydroxylated by prolyl hydroxylase (PHD). Hydroxylation is promoted by von Hippel-Lindau tumor suppressor protein binding to HIF- $1 \alpha$ subunit. As a result, HIF- $1 \alpha$ subunit is destroyed by proteasome. As PHD activity is decreased under hypoxic condition, HIF- $1 \alpha$ subunit heterodimerizes with the constitutively expressed HIF- $1 \beta$ subunit. The heterodimeric HIF translocates into the nucleus, activating gene transcriptions, such as angiogenesis, cell metabolism, cell growth, apoptosis, and so on [16]. However, it has been reported that activation of HIF becomes less responsive to renal hypoxia in advanced renal dysfunction $[17,18]$. It has also been reported that oral administration of cobalt chloride, which activates HIF, ameliorates renal injury in diabetic nephropathy of SHR/NDmercp rats [7], and that VEGF plays an important role in capillary repair in damaged glomeruli in glomerulonephritis rats induced by injection of anti-Thy-1.1 antibody [8]. On the basis of these reports, it is suggested that the treatments against hypoxia are useful for suppressing the progression of CKD.

The 5/6 nephrectomized rats we used in this study are a typical model of progressive renal disease. The initial change in this model causes increasing glomerular capillary pressure associated with a relative decrease in the expansion of efferent arterioles, resulting in glomerular hyperfiltration and hypertension. Glomerular hyperfiltration activates the renin-angiotensin system, eventually leading to glomerular sclerosis [19]. However, it has been reported recently that the deterioration of renal function in this model is correlated with an interstitial damage rather than a glomerular damage, and that chronic tissue hypoxia due to the initial reduction of blood flow causes the progression of renal failure [12]. Furthermore, it has been reported that the continuous infusion of dimethyloxalylglycine (DMOG), an activator of HIF, suppressed the increase of proteinuria in this model [20].

There have been some reports regarding the protective effects of hachimijiogan on renal function. Hachimijiogan had antihypertensive and renal-protective effects on Dahl salt-sensitive hypertensive rats, and its mechanism was suspected to enhance the production of prostaglandin $E_{2}$ [21]. Hachimijiogan had protective effects on diabetic nephropathy rats induced by streptozotocin injection, with its mechanism being suspected of improving lipid metabolism, glucose metabolism, and oxidative stress [22]. Hachimijiogan had renal-protective effects by improving oxidative stress and suppressing expression of fibronectin and TGF- $\beta$ in spontaneously diabetic nephropathy rats [10]. Hachimijiogan also had renal-protective effects on 5/6 nephrectomized rats by a mechanism thought to reduce uremic toxins associated with oxidative stress [11]. Morroniside is a main component of Corni Fructus, which is contained in hachimijiogan, and was reported to have a renal-protective effect on diabetic 


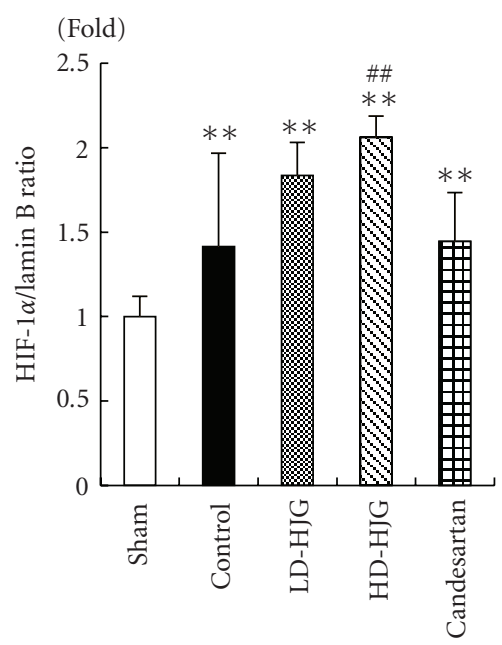

(a)

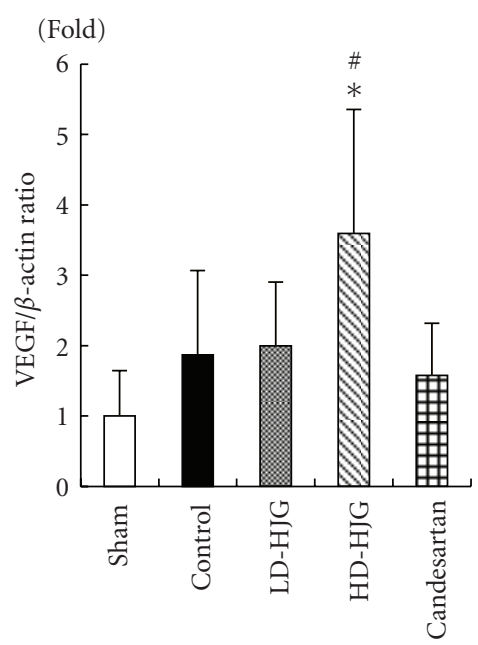

(b)

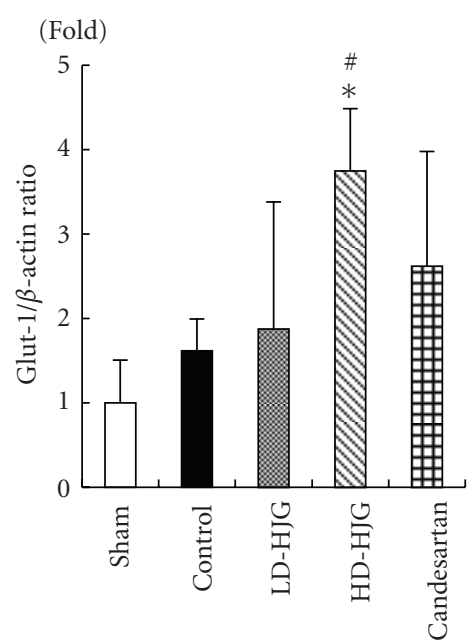

(c)

Figure 2: Measurement of renal cortical hypoxia-related factors in remnant kidney treated with hachimijiogan. The volume of HIF-1 $\alpha$ protein of the four $5 / 6 \mathrm{Nx}$ groups increased significantly compared to that of the sham group, and that of the high-dose hachimijiogan group increased significantly compared to that of the control group (a). The mRNA levels of VEGF and Glut-1 of the high-dose hachimijiogan group increased significantly compared to those of the sham and control groups (b, c). (a) HIF-1 $\alpha$ protein, (b) VEGF mRNA, (c) Glut-1 mRNA, LD-HJG: low-dose hachimijiogan, HD-HJG: high-dose hachimijiogan. Data represent mean \pm S.D. $(n=8-10) .{ }^{*} P<.05,{ }^{* *} P<.01$ versus sham group, ${ }^{\#} P<.05,{ }^{\#} P<.01$ versus control group.

nephropathy rats by inhibiting the production of advanced glycation end product and oxidative stress [23].

In this study, the levels of BUN, s-Cre, and urinary protein excretion of the control group were significantly higher than those of the sham group whereas the level of Ccr of the control group significantly decreased compared to that of the sham group. The HIF-1 $\alpha$ protein level of the control group increased compared to that of the sham group one week after nephrectomy, as previously reported [12].

On the other hand, administration of high-dose hachimijiogan significantly reduced urinary protein excretion and elevated the HIF- $1 \alpha$ protein level in the renal cortex. Therefore, it was suggested that hachimijiogan had an activating effect on HIF. This was also supported by the observations of increased VEGF mRNA and Glut-1 mRNA. Immunohistological examination of renal tubular epithelial cells also showed an increase in HIF- $1 \alpha$ by the administration of hachimijiogan.

Administration of candesartan cilexetil, which has renalprotective effects $[24,25]$, significantly decreased urinary protein excretion. However, the HIF- $1 \alpha$ protein level and the expressions of VEGF mRNA and Glut 1 mRNA did not increase by candesartan cilexetil administration. It had been reported that HIF activation in renal tissue decreases by the administration of candesartan cilexetil [12]. It is supposed that the main mechanism contributing to the attenuation of proteinuria by the administration of ARB is a reduction in glomerular capillary pressure, as previously reported [26].

It has already been reported that oxidative stress increased in patients with CKD [27]. In the state of uremia, due to the increase of reactive oxygen species in the vascular endothelium, a reduction in glutathione [28] and an increase in releasing reactive oxygen species from leukocytes [29] have been reported. It has also been reported that the activation of the renin-angiotensin system suppresses the expression of super oxide dismutase (SOD) [30]. This oxidative stress has been reported to have the possibility of inhibiting the activation of HIF [31]. Therefore, the involvement of oxidative stress was examined by measuring urinary 8OHdG. The results showed that, compared with the sham group, the control group had a significantly increased level of urinary $8-\mathrm{OHdG}$, and the hachimijiogan and candesartan cilexetil groups had significantly decreased levels of urinary 8-OHdG. The antioxidant effects of hachimijiogan have been reported $[10,11]$. Although ARB has also been reported to have antioxidant activity [32], the HIF activation in the candesartan cilexetil group did not increase in this study. Therefore, the mechanism of increasing HIF of the hachimijiogan group was considered to be a direct effect of hachimijiogan.

There are various reports about natural products that have an effect on HIF activation [33]. Concerning phenolic compounds, quercetin, contained in red wine, has been reported to have the effect of HIF activation on cultured murine brain endothelial cells in normoxia [34]. Green tea extract and its major component epigallocatechin gallate (EGCG) have been reported to activate HIF in human prostate cancer cells (PC-3ML) by inhibiting the degradation with PHD [35]. However, EGCG has also been reported to have a suppressive effect on HIF activation in HeLa cells and HepG2 cells by inhibiting the PI3K-AKT-mTOR pathway [36]. It is suggested that the activations of HIF and VEGF depend on the kinds of cultured cells or culture conditions. Moreover, there are as yet few reports on the study of the activation of HIF in vivo. 


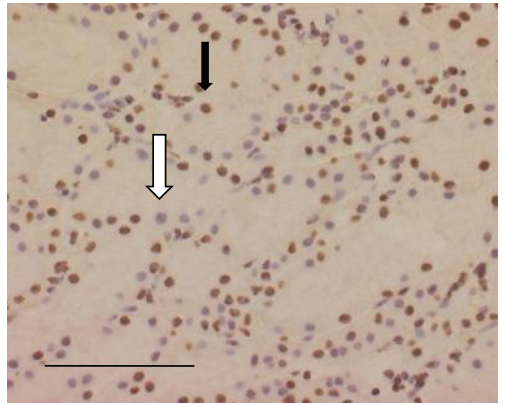

(a)

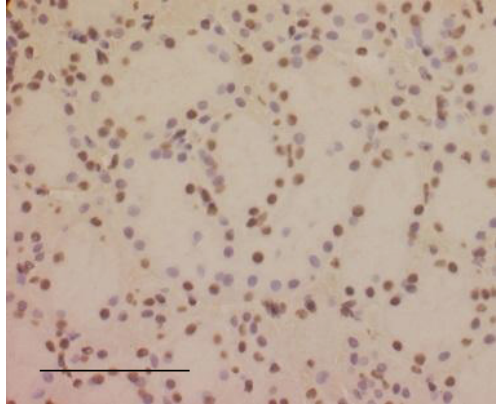

(b)

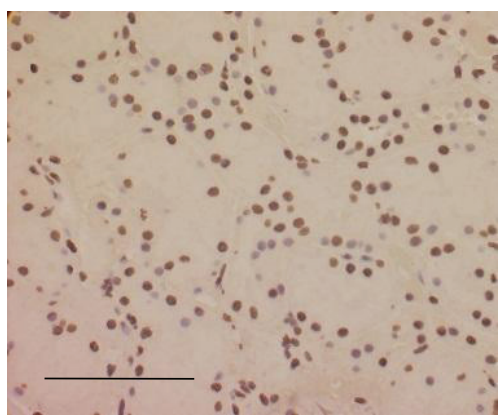

(c)

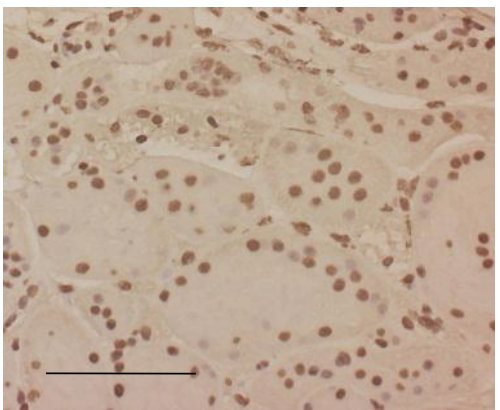

(d)

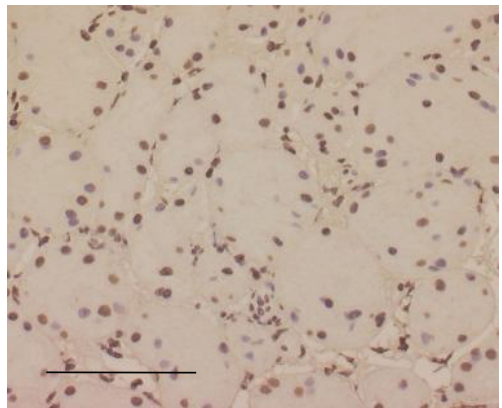

(e)

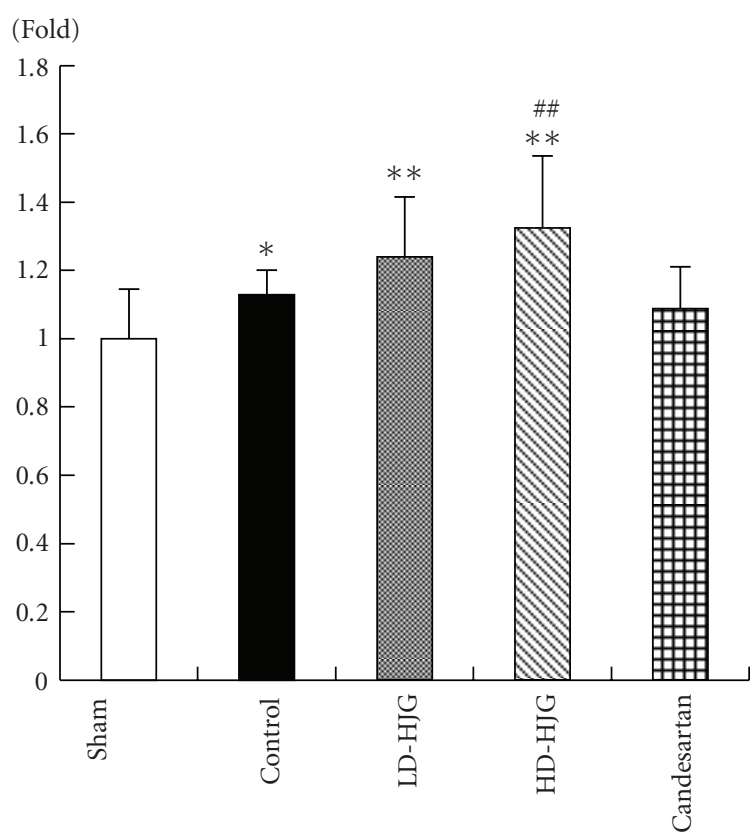

(f)

Figure 3: Immunohistochemical studies showed that the expression of HIF- $1 \alpha$ of the high-dose hachimijiogan group increased compared to that of the control group (a-e). The black arrow indicates an HIF- $1 \alpha$-positive cell stained brown, and the white arrow indicates an HIF$1 \alpha$-negative cell stained blue. The number of HIF- $1 \alpha$-positive cells in the high-dose hachimijiogan group increased significantly compared to that in the control group. (a) sham, (b) control, (c) low-dose hachimijiogan, (d) high-dose hachimijiogan, (e) candesartan cilexetil. (magnification 400x, scale bar: $100 \mu \mathrm{m}$ ), (f) Comparison with percentage of HIF-1 $\alpha$-positive cells/field in the every group. Data represent mean \pm S.D. ${ }^{*} P<.05,{ }^{* *} P<.01$ versus sham group, ${ }^{\# \#} P<.01$ versus control group. 


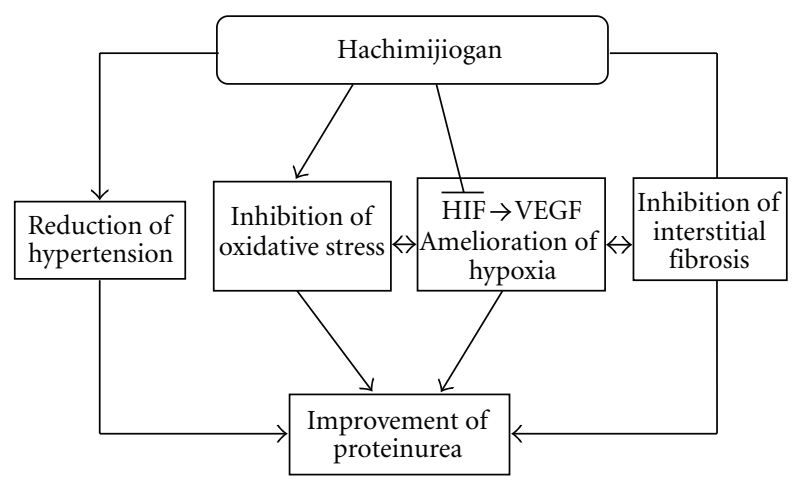

FIGURE 4: Hypothetical representation of the effects of hachimijiogan on renal dysfunction.

\section{Conclusion}

It has been suggested that hachimijiogan has a renalprotective effect through the influence of HIF activation. We summarized our hypothetical representation that explains the effects of hachimijiogan on renal dysfunction in Figure 4. Further experiments are needed to determine the active components and the mechanisms of the activation of HIF, and to study the long-term effects of hachimijiogan in vivo. Meanwhile, in terms of renal treatment via HIF, the hachimijiogan treatment is considered as a new therapeutic tool in addition to the existing renal treatments.

\section{Acknowledgments}

The authors are grateful to Mr. Arndt Gerz and Mr. Thomas Fenwick for assisting with the English version of the paper. This paper was supported by a Grant-in-Aid for Scientific Research (C) (no. 21590758) by the Japan Society for the Promotion of Science (JSPS).

\section{References}

[1] A. S. Go, G. M. Chertow, D. Fan, C. E. McCulloch, and C. Y. Hsu, "Chronic kidney disease and the risks of death, cardiovascular events, and hospitalization," New England Journal of Medicine, vol. 351, no. 13, pp. 1296-1305, 2004.

[2] M. J. Lysaght, "Maintenance dialysis population dynamics: current trends and long-term implications," Journal of the American Society of Nephrology, vol. 13, supplement 1, pp. S37-S40, 2001.

[3] G. Remuzzi and T. Bertani, "Pathophysiology of progressive nephropathies," New England Journal of Medicine, vol. 339, no. 20, pp. 1448-1456, 1998.

[4] L. G. Fine, D. Bandyopadhay, and J. T. Norman, "Is there a common mechanism for the progression of different types of renal diseases other than proteinuria? Towards the unifying theme of chronic hypoxia," Kidney International, Supplement, vol. 57, no. 75, pp. S22-S26, 2000.

[5] K. U. Eckardt, C. Rosenberger, J. S. Jürgensen, and M. S. Wiesener, "Role of hypoxia in the pathogenesis of renal disease," Blood Purification, vol. 21, no. 3, pp. 253-257, 2003.

[6] J. Marx, "How cells endure low oxygen," Science, vol. 303, no. 5663, pp. 1454-1456, 2004.
[7] S. Ohtomo, M. Nangaku, Y. Izuhara, S. Takizawa, C. Y. Strihou, and T. Miyata, "Cobalt ameliorates renal injury in an obese, hypertensive type 2 diabetes rat model," Nephrology Dialysis Transplantation, vol. 23, no. 4, pp. 1166-1172, 2008.

[8] Y. Masuda, A. Shimizu, T. Mori et al., "Vascular endothelial growth factor enhances glomerular capillary repair and accelerates resolution of experimentally induced glomerulonephritis," American Journal of Pathology, vol. 159, no. 2, pp. 599608, 2001.

[9] K. Terasawa, "Kampo: Japanese-Oriental Medicine, insights from clinical cases," K.K. Standard McIntyre, pp. 243-244, 1993.

[10] T. Nakagawa, T. Yokozawa, N. Yamabe et al., "Long-term treatment with Hachimi-jio-gan attenuates kidney damage in spontaneously diabetic WBN/Kob rats," Journal of Pharmacy and Pharmacology, vol. 57, no. 9, pp. 1205-1212, 2005.

[11] N. Yamabe, T. Yokozawa, H. Y. Kim, and E. J. Cho, "Protective effect of Hachimi-jio-gan against renal failure in a subtotal nephrectomy rat model," Journal of Pharmacy and Pharmacology, vol. 57, no. 12, pp. 1637-1644, 2005.

[12] K. Manotham, T. Tanaka, M. Matsumoto et al., "Evidence of tubular hypoxia in the early phase in the remnant kidney model," Journal of the American Society of Nephrology, vol. 15, no. 5, pp. 1277-1288, 2004.

[13] T. Tanaka, I. Kojima, T. Ohse et al., "Cobalt promotes angiogenesis via hypoxia-inducible factor and protects tubulointerstitium in the remnant kidney model," Laboratory Investigation, vol. 85, no. 10, pp. 1292-1307, 2005.

[14] T. Kumada, K. Tsuneyama, H. Hatta, S. Ishizawa, and Y. Takano, "Improved 1-h rapid immunostaining method using intermittent microwave irradiation: practicability based on 5 years application in Toyama Medical and Pharmaceutical University Hospital," Modern Pathology, vol. 17, no. 9, pp. 1141-1149, 2004.

[15] M. Nangaku and K. U. Eckardt, "Hypoxia and the HIF system in kidney disease," Journal of Molecular Medicine, vol. 85, no. 12, pp. 1325-1330, 2007.

[16] R. H. Wenger, D. P. Stiehl, and G. Camenisch, "Integration of oxygen signaling at the consensus HRE," Science's STKE, vol. 2005, no. 306, p. re12, 2005.

[17] P. Katavetin, T. Miyata, R. Inagi et al., "High glucose blunts vascular endothelial growth factor response to hypoxia via the oxidative stress-regulated hypoxia-inducible factor/hypoxiaresponsible element pathway," Journal of the American Society of Nephrology, vol. 17, no. 5, pp. 1405-1413, 2006.

[18] P. Katavetin, R. Inagi, T. Miyata et al., "Albumin suppresses vascular endothelial growth factor via alteration of hypoxiainducible factor/hypoxia-responsive element pathway," Biochemical and Biophysical Research Communications, vol. 367, no. 2, pp. 305-310, 2008.

[19] T. H. Hostetter, J. L. Olson, H. G. Rennke, M. A. Venkatachalam, and B. M. Brenner, "Hyperfiltration in remnant nephrons: a potentially adverse response to renal ablation," Journal of the American Society of Nephrology, vol. 12, no. 6, pp. 1315-1325, 2001.

[20] Y. R. Song, S. J. You, Y. M. Lee et al., "Activation of hypoxiainducible factor attenuates renal injury in rat remnant kidney," Nephrology Dialysis Transplantation, vol. 25, no. 1, pp. 77-85, 2010.

[21] N. Hirawa, Y. Uehara, Y. Kawabata et al., "Hachimi-jio-gan extract protects the kidney from hypertensive injury in dahl salt-sensitive rat," American Journal of Chinese Medicine, vol. 24, no. 3-4, pp. 241-254, 1996. 
[22] T. Yokozawa, N. Yamabe, E. J. Cho, T. Nakagawa, and S. Oowada, "A study on the effects to diabetic nephropathy of hachimi-jio-gan in rats," Nephron Experimental Nephrology, vol. 97, no. 2, pp. e38-e48, 2004.

[23] N. Yamabe, K. S. Kang, E. Goto, T. Tanaka, and T. Yokozawa, "Beneficial effect of Corni Fructus, a constituent of Hachimijio-gan, on advanced glycation end-product-mediated renal injury in streptozotocin-treated diabetic rats," Biological and Pharmaceutical Bulletin, vol. 30, no. 3, pp. 520-526, 2007.

[24] K. Ueshima, S. Yasuno, K. Oba et al., "Impact of left ventricular hypertrophy on the time-course of renal function in hypertensive patients: a subanalysis of the CASE-J trial," Circulation Journal, vol. 74, no. 10, pp. 2132-2138, 2010.

[25] P. Morsing, "Candesartan: a new-generation angiotensin II AT receptor blocker: pharmacology, antihypertensive efficacy, renal function, and renoprotection," Journal of the American Society of Nephrology, vol. 10, supplement 11, pp. S248-S254, 1999.

[26] H. Rincon-Choles, B. S. Kasinath, Y. Gorin, and H. E. Abboud, "Angiotensin II and growth factors in the pathogenesis of diabetic nephropathy," Kidney International, Supplement, vol. 62, no. 82, pp. S8-S11, 2002.

[27] E. Dounousi, E. Papavasiliou, A. Makedou et al., "Oxidative stress is progressively enhanced with advancing stages of CKD," American Journal of Kidney Diseases, vol. 48, no. 5, pp. 752-760, 2006.

[28] L. Dou, N. Jourde-Chiche, V. Faure et al., "The uremic solute indoxyl sulfate induces oxidative stress in endothelial cells," Journal of Thrombosis and Haemostasis, vol. 5, no. 6, pp. 13021308, 2007.

[29] S. Sela, R. Shurtz-Swirski, M. Cohen-Mazor et al., "Primed peripheral polymorphonuclear leukocyte: a culprit underlying chronic low-grade inflammation and systemic oxidative stress in chronic kidney disease," Journal of the American Society of Nephrology, vol. 16, no. 8, pp. 2431-2438, 2005.

[30] W. J. Welch, J. Blau, H. Xie, T. Chabrashvili, and C. S. Wilcox, "Angiotensin-induced defects in renal oxygenation: role of oxidative stress," American Journal of Physiology, vol. 288, no. 1, pp. H22-H28, 2005.

[31] C. Rosenberger, M. Khamaisi, Z. Abassi et al., "Adaptation to hypoxia in the diabetic rat kidney," Kidney International, vol. 73, no. 1, pp. 34-42, 2008.

[32] T. W. Kurtz and M. Pravenec, "Antidiabetic mechanisms of angiotensin-converting enzyme inhibitors and angiotensin II receptor antagonists: beyond the renin-angiotensin system," Journal of Hypertension, vol. 22, no. 12, pp. 2253-2261, 2004.

[33] D. G. Nagle and Y. D. Zhou, "Natural product-derived small molecule activators of hypoxia-inducible factor-1 (HIF-1)," Current Pharmaceutical Design, vol. 12, no. 21, pp. 2673-2688, 2006.

[34] W. J. Wilson and L. Poellinger, "The dietary flavonoid quercetin modulates HIF- $1 \alpha$ activity in endothelial cells," Biochemical and Biophysical Research Communications, vol. 293, no. 1, pp. 446-450, 2002.

[35] R. Thomas and M. H. Kim, "Epigallocatechin gallate inhibits HIF- $1 \alpha$ degradation in prostate cancer cells," Biochemical and Biophysical Research Communications, vol. 334, no. 2, pp. 543548, 2005.

[36] Q. Zhang, X. Tang, Q. Lu, Z. Zhang, J. Rao, and A. D. Le, "Green tea extract and (-)-epigallocatechin-3-gallate inhibit hypoxia- and serum-induced HIF-1 protein accumulation and VEGF expression in human cervical carcinoma and hepatoma cells," Molecular Cancer Therapeutics, vol. 5, no. 5, pp. 1227$1238,2006$. 


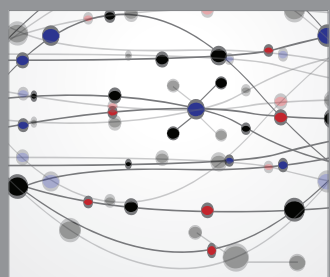

The Scientific World Journal
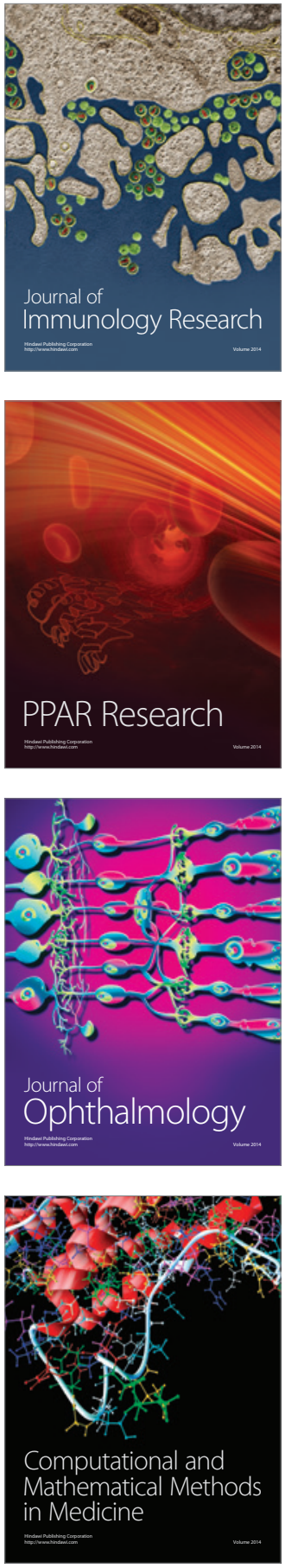

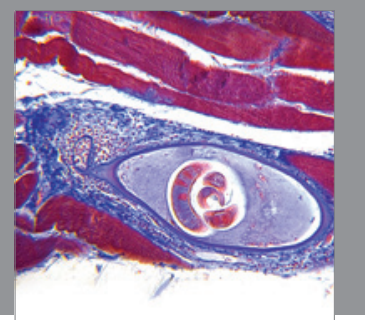

Gastroenterology

Research and Practice
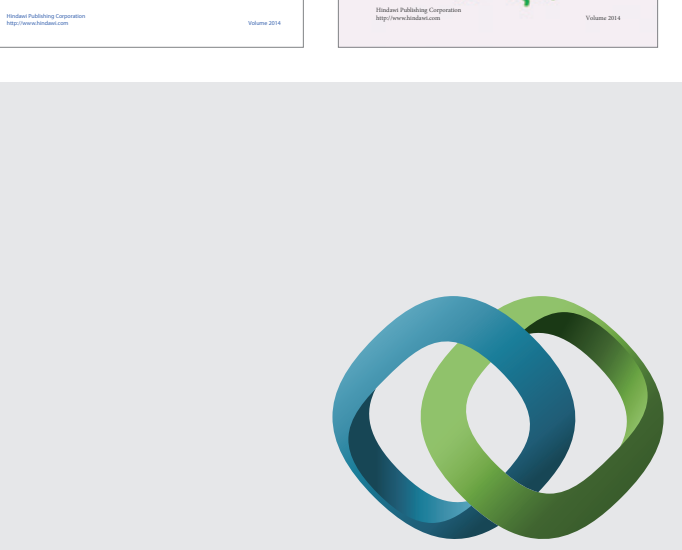

\section{Hindawi}

Submit your manuscripts at

http://www.hindawi.com
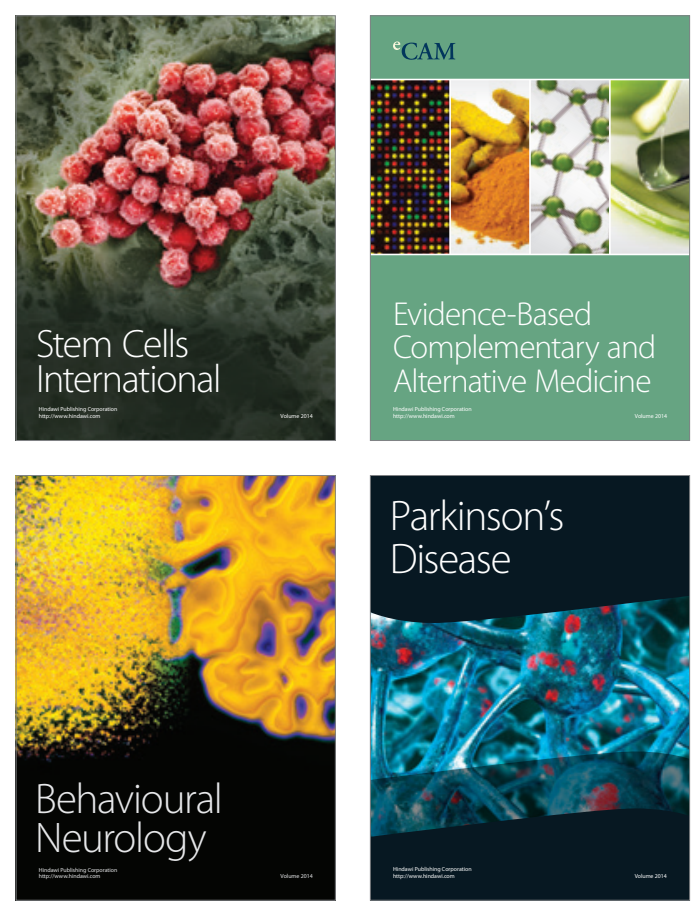

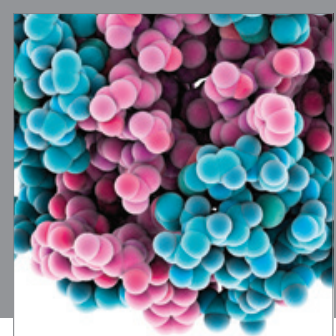

Journal of
Diabetes Research

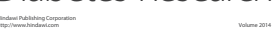

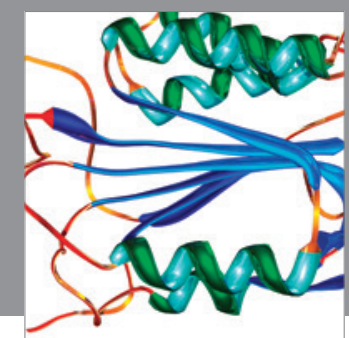

Disease Markers
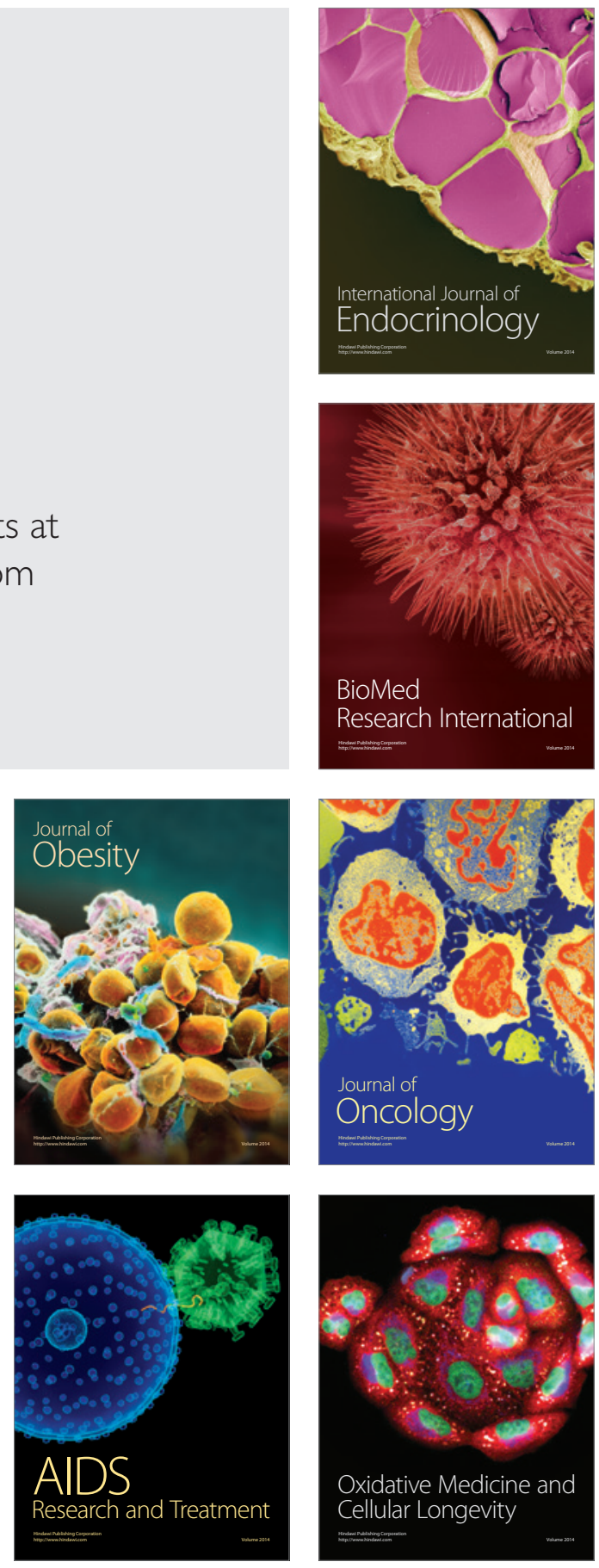